\title{
DISCRETE LINEAR WEINGARTEN SURFACES WITH SINGULARITIES IN RIEMANNIAN AND LORENTZIAN SPACEFORMS
}

\author{
WAYNE ROSSMAN AND MASASHI YASUMOTO
}

\begin{abstract}
In this paper we define and analyze singularities of discrete linear Weingarten surfaces with Weierstrass-type representations in 3-dimensional Riemannian and Lorentzian spaceforms. In particular, we discuss singularities of discrete surfaces with non-zero constant Gaussian curvature, and parallel surfaces of discrete minimal and maximal surfaces, and discrete constant mean curvature 1 surfaces in de Sitter 3 -space, including comparisons with different previously known definitions of such singularities.
\end{abstract}

\section{INTRODUCTION}

In this paper we examine discrete surfaces with Weierstrass-type representations in spaceforms, taking advantage of the more general setting of Lie sphere geometry and discrete Legendre immersions (see Definition 2.1 here), and with helpful motivations coming from the developing field of $\Omega$ surfaces. There are numerous Weierstrass-type representations in 3-dimensional spaceforms in addition to the classical representation for minimal surfaces in $\mathbb{R}^{3}$, for example, for

(1) maximal surfaces (spacelike immersion with mean curvature identically 0 ) in Minkowski 3 -space $\mathbb{R}^{2,1}$ by Kobayashi [22,

(2) constant mean curvature (CMC, for short) 1 surfaces in hyperbolic 3-space $\mathbb{H}^{3}$ by Bryant [6] (see also [29]),

(3) flat surfaces in $\mathbb{H}^{3}$ by Gálvez, Martínez, Milán [14,

(4) CMC 1 surfaces in de Sitter 3-space $\mathbb{S}^{2,1}$ by Aiyama, Akutagawa [1],

(5) linear Weingarten surfaces of Bryant type (BrLW surfaces, for short) in $\mathbb{H}^{3}$ by Gálvez, Martínez, Milán [15],

(6) linear Weingarten surfaces of Bianchi type (BiLW surfaces, for short) in $\mathbb{S}^{2,1}$ by Aledo, Espinar [2].

Regarding the last two examples above, Izumiya and Saji [20] showed that a necessary and sufficient condition for an immersion in $\mathbb{H}^{3}$ to be BrLW is that its unit normal vector field is BiLW (see $\$ 4$ ).

Recently, there has been work on discretization of the above representations. Bobenko, Pinkall [3] described discrete isothermic surfaces in the Euclidean 3-space $\mathbb{R}^{3}$, and as an application, they derived the Weierstrass representation for discrete isothermic minimal surfaces in $\mathbb{R}^{3}$, using integrable systems techniques. In the same vein, Hertrich-Jeromin

2010 Mathematics Subject Classification. Primary 53A10, Secondary 52C99.

Key words and phrases. discrete differential geometry, surface theory, integrable systems. 
[16] gave the Weierstrass-type representation for discrete isothermic CMC 1 surfaces in $\mathbb{H}^{3}$.

Burstall, Hertrich-Jeromin and the first author [9] described discrete linear Weingarten surfaces in any 3-dimensional spaceform using Lie sphere geometry, which we briefly introduce in \$5. Using that method, we can treat discrete linear Weingarten surfaces in any 3-dimensional spaceform. They did not consider singularities of discrete surfaces, however, as we will do here.

Returning to smooth surfaces, unlike the minimal and (non-zero) CMC surfaces in $\mathbb{R}^{3}$, general linear Weingarten surfaces will have singularities. In fact, singularities of maximal surfaces in $\mathbb{R}^{2,1}$, flat and BrLW surfaces in $\mathbb{H}^{3}$, and BiLW surfaces in $\mathbb{S}^{2,1}$ are investigated in [13, 23, 30]. Thus, it is natural to still consider singularities when discretizing these surfaces. However, difficulties occur with this (Definition 3.1), and overcoming those difficulties is our primary task here.

Hoffmann, Sasaki, Yoshida and the first author [19] described discrete BrLW surfaces in $\mathbb{H}^{3}$, and furthermore treated singularities of discrete flat surfaces in $\mathbb{H}^{3}$. For that, they considered the behavior of caustics of smooth flat surfaces at a singular point, via the Weierstrass-type representation. Such a caustic contacts the surface at a singular point, which lead to a natural definition of singularities in the discrete case, i.e. that a singularity of a discrete flat surface is a vertex that contacts the (discrete) caustic. We will define singular vertices in a more direct way that applies to a wider variety of discrete surfaces, and show equivalence of the definitions in the case considered just above (Theorem 6.1).

The second author 32 described discrete maximal surfaces in the Minkowski 3-space $\mathbb{R}^{2,1}$ and analyzed their singular faces, that is, non-spacelike faces (Definition 8.1). This is also a natural way to define singular behavior, because the tangent plane of a smooth maximal surface is non-spacelike precisely at singular points.

Thus, singularities of discrete surfaces could be either vertices or faces, and two of our primary results here are about relating those two viewpoints, in particular, in the cases of discrete maximal surfaces in $\mathbb{R}^{2,1}$ and discrete CMC 1 surfaces in $\mathbb{S}^{2,1}$.

Smooth 2-dimensional Legendre immersions in Lie sphere geometry project to surfaces in spaceforms that can have singularities. However, those surfaces considered together with their unit normal maps become immersions (by definition), and they are called fronts. The most typical singularities on fronts are cuspidal edges of $3 / 2$ type, and next perhaps are swallowtails. At such singularities, exactly one of the principal curvatures will diverge (see [28]), and equivalently, one of the principal curvature spheres will become a point sphere. Using that the notion of principal curvature spheres in Lie sphere geometry is independent of the choice of projection to a 3-dimensional spaceform, we define singular vertices on projections of discrete Legendre immersions.

While typical singularities on smooth surfaces can be found by locating the points where one principal curvature blows out to infinity, on discrete surfaces the principal curvatures are discrete functions from the set of edges to the real numbers, and thus we can only identify the vertices at which a principal curvature changes sign. As a result, it is not so immediate to distinguish the points that are singular from the points that are parabolic (at which exactly one of the two bounded principal curvature becomes zero) or flat (at which both principal curvatures become zero). This is why we will use 
a particular terminology "FPS vertices" in Definition 3.1. This is the first of our three goals:

(1) We will find and examine cases where the distinction between singular and parabolic or flat points is possible. Such cases include surfaces of constant Gaussian curvature (CGC) $K \neq 0$ (see $\$ 6$ ), and some particular discrete linear Weingarten surfaces for which Weierstrass type representations exist (\$7, \$8, $\$ 91$.

(2) We will confirm that the discrete Weierstrass type representations are compatible with other ways of defining discrete surfaces with specific curvature properties. In particular, they are compatible with the definitions given by Burstall, Hertrich-Jeromin and the first author in [9] (Proposition 5.1).

(3) We will find relationships between singular vertices and singular faces in particular cases (Theorem 8.1, Theorem 9.1, Corollary 9.1).

\section{Discrete Legendre immersions}

First we recall smooth Legendre immersions in the context of Lie sphere geometry, that is, maps $\Lambda$ of 2 -manifolds $M^{2}$ into the collection of null planes in $\mathbb{R}^{4,2}$, with metric signature $(-,+,+,+,+,-)$, i.e.

$$
(X, Y)_{\mathbb{R}^{4,2}}=(X, Y):=-x_{1} y_{1}+x_{2} y_{2}+x_{3} y_{3}+x_{4} y_{4}+x_{5} y_{5}-x_{6} y_{6}
$$

for $X=\left(x_{1}, x_{2}, x_{3}, x_{4}, x_{5}, x_{6}\right)^{t}, Y=\left(y_{1}, y_{2}, y_{3}, y_{4}, y_{5}, y_{6}\right)^{t} \in \mathbb{R}^{4,2}$. Then

$$
\mathbb{L}^{5}:=\left\{X \in \mathbb{R}^{4,2} \mid(X, X)=0\right\}
$$

denotes the light cone of $\mathbb{R}^{4,2}$.

Let $\Lambda \subset \mathbb{L}^{5}$ be a 2-dimensional null subspace, which projectivizes to a line in the projectivized light cone $\mathbb{P L}^{5}$ called a contact element. This line will represent a family of spheres (a pencil) that are all tangent (with same orientation) at one point.

If $\Lambda$ is a (smooth) map from $M=M^{2}$ to the collection of null planes in $\mathbb{R}^{4,2}$, where $M$ is a 2-dimensional manifold, then $\Lambda$ is a Legendre immersion if,

(1) for any pair of sections $X_{1}, X_{2}$ of $\Lambda$,

$$
d X_{1} \perp X_{2} \quad \text { (contact condition), and }
$$

(2) for any $m \in M$ and any choice of $Y \in T_{m} M, d X(Y) \in \Lambda(m)$ for all sections $X$ of $\Lambda(m)$ implies $Y=0$ (immersion condition).

The immersion condition can be restated in terms of a basis of sections for the null planes $\Lambda$ as follows: If

$$
\Lambda=\operatorname{span}\left\{X_{1}, X_{2}\right\}
$$

with basis $X_{1}, X_{2}: M^{2} \rightarrow \mathbb{L}^{5}$, then the immersion condition is equivalent to

$$
d X_{1}(Y), d X_{2}(Y) \in \Lambda(m) \text { implies } Y=0
$$

for all $Y \in T_{m} M$, and one can then check that this condition is independent of the choice of basis $X_{1}, X_{2}$.

By choosing two nonzero perpendicular vectors $p, q$ in $\mathbb{R}^{4,2}$ ( $p$ not null), we can project $\Lambda$ to a surface $f: M^{2} \rightarrow M^{3}$ in the 3 -dimensional spaceform

$$
M^{3}=M_{p, q}^{3}:=\left\{X \in \mathbb{R}^{4,2} \mid(X, X)=(X, p)=0,(X, q)=-1\right\}
$$


with sectional curvature $-(q, q)$, by taking $f \in \operatorname{Sec}(\Lambda)$ such that

$$
(f, p)=0 \text { and }(f, q)=-1 \text {, }
$$

where $\operatorname{Sec}(\Lambda)$ denotes the set of all sections of $\Lambda$. Note that, when we choose a constant timelike (resp. spacelike) vector $p \in \mathbb{R}^{4,2}$ and a constant vector $q \in \mathbb{R}^{4,2}, M^{3}$ becomes a 3-dimensional Riemannian (resp. Lorentzian) spaceform. For details, see [26], and for a particular choice of $p$ and $q$, see Section 4 .

Let $n$ denote the unit normal to $f$ in $M^{3}$, i.e. $n \in \operatorname{Sec}(\Lambda)$ and

$$
(n, q)=0 \text { and }(n, p)=-1 .
$$

The sections of $\Lambda=\operatorname{span}\{f, n\}$ represent the sphere congruences of $f$, and then $f$, resp. $n$, is the point sphere, resp. tangent geodesic plane, congruence. Let $s_{\alpha}$ for $\alpha=1,2$ be sections of $\Lambda$ that represent the principal curvature sphere congruences, which can be defined by

$$
s_{\alpha}=\kappa_{\alpha} f+n
$$

using the principle curvatures $\kappa_{\alpha}$ of $f$, or equivalently by the directional derivative conditions that $D_{\vec{v}_{\alpha}} s_{\alpha} \in \operatorname{Sec}(\Lambda)$ for some tangent vector fields $\vec{v}_{\alpha}$ on $M^{2}$.

For $\Lambda$ above to be a Legendre immersion, both immersion and contact conditions must be satisfied. For a discrete Legendre map $\Lambda$ as in Definition 2.1 below, discretized versions of the immersion and contact conditions are needed. We also assume the existence of "discrete curvature line coordinates", that is, we require that the four vertices of each quadrilateral be concircular, which is called a principal net. In this way, the properties of smooth Legendre immersions motivate the following definition of discrete Legendre immersions:

Definition $2.1([9])$. A map

$$
\left.\Lambda: \mathbb{Z}^{2} \text { (or some subdomain of } \mathbb{Z}^{2}\right) \rightarrow\left\{\text { null planes in } \mathbb{R}^{4,2}\right\}
$$

is a discrete Legendre immersion if, for any quadrilateral, with vertices $i, j, k$, $\ell$ ordered counterclockwise about the quadrilateral and with $i$ in the lower left corner in $\mathbb{Z}^{2}$, and with corresponding surface vertices $f_{i}, f_{j}, f_{k}, f_{l}$ defined like in (1),

(1) (principal net condition) $\operatorname{dim}\left(\operatorname{span}\left\{f_{i}, f_{j}, f_{k}, f_{\ell}\right\}\right)=3$,

(2) (first immersion condition) There exist $p, q$ such that the difference of any two of $f_{i}, f_{j}, f_{k}, f_{\ell}$ is non-null,

(3) (second immersion condition) For some $p, q$ as in item (2) above, $f_{k}-f_{i}$ and $f_{\ell}-f_{j}$ are not parallel,

(4) (contact condition) $\Lambda_{i} \cap \Lambda_{j} \neq\{\overrightarrow{0}\}, \Lambda_{i} \cap \Lambda_{\ell} \neq\{\overrightarrow{0}\}$.

Remark. Item (1) in Definition 2.1 and $\left(f_{*}, q\right)=-1$ imply

$$
f_{i}, f_{j}, f_{k}, f_{\ell}
$$

all lie in some 2-dimensional plane. Item (3) implies any two or three vertices amongst $f_{i}, f_{j}, f_{k}, f_{\ell}$ span a 2 or 3 dimensional subspace of $\mathbb{R}^{4,2}$, respectively, with nondegenerate induced metric $(+,-)$ or $(+,+,-)$. 


\section{FPS VERTICES OF PROJECTIONS OF DISCRETE LEGENDRE IMMERSIONS}

Generically, a smooth surface (section) $f \in \operatorname{Sec}(\Lambda)$ will have a singularity when one of the principal curvature spheres $s_{\alpha}$ becomes a point sphere [28], i.e. when $s_{\alpha} \perp p$ for $\alpha=1$ or 2 . Also, where $f$ does not have a singularity, it will have a parabolic or flat point if one of the $s_{\alpha}$ becomes a tangent geodesic plane, i.e. $s_{\alpha} \perp q$.

In the case of discrete Legendre immersions, the domain becomes $\mathbb{Z}^{2}$, or some subdomain of $\mathbb{Z}^{2}$, rather than $M^{2}$. We define the curvature spheres as those spheres represented by nonzero vectors $([9])$

$$
s_{1} \in \Lambda_{i} \cap \Lambda_{j} \text { and } s_{2} \in \Lambda_{i} \cap \Lambda_{\ell} .
$$

Thus we have spheres in $M^{3}$, associated to edges, that lie in both of the sphere pencils defined at the two endpoints of the edges. In particular the normal geodesics (i.e. the geodesics through the vertices and perpendicular to the spheres in the sphere pencils) emanating from the adjacent vertices, when they do intersect, will intersect at equal distances from the two vertices.

Thus, $s_{1}=s_{(m, n),(m+1, n)}$ will be defined on horizontal edges from vertex $i=(m, n) \in$ $\mathbb{Z}^{2}$ to vertex $j=(m+1, n) \in \mathbb{Z}^{2}$ as the representative (for a sphere) that is common to both the null planes $\Lambda_{i}$ and $\Lambda_{j}$, and $s_{2}=s_{(m, n),(m, n+1)}$ is defined analogously on vertical edges from $i$ to $\ell=(m, n+1)$. We then define the principal curvatures by ([4], [9])

$$
\kappa_{i j}=\frac{\left(s_{1}, q\right)}{\left(s_{1}, p\right)}, \quad \kappa_{i \ell}=\frac{\left(s_{2}, q\right)}{\left(s_{2}, p\right)} .
$$

As the principal curvature spheres $s_{\alpha}$ and principal curvatures $\kappa_{\alpha \beta}$ are defined on edges, not vertices, we lose the ability to look for points in the domain where $s_{\alpha}$ is exactly perpendicular to $p$ or $q$. Thus we reformulate the conditions for singularities and parabolic or flat points by finding vertices in the domain at which the $\kappa_{\alpha \beta}$ change sign in at least one direction:

Definition 3.1. For a $\Lambda$ as in Definition 2.1, together with a choice of spaceform determined from a choice of $p$ and $q$, we say that $(m, n)$ is a flat-or-parabolic-or-singular (FPS) vertex if

$$
\kappa_{(m-1, n),(m, n)} \cdot \kappa_{(m, n),(m+1, n)} \leq 0 \quad \text { or } \quad \kappa_{(m, n-1),(m, n)} \cdot \kappa_{(m, n),(m, n+1)} \leq 0 .
$$

When both $p$ and $q$ are non-null, switching $p$ and $q$ will result in the projected surface $f$ changing to its Gauss map $n$. In the smooth case, generically, a parabolic or flat point on one of the two surfaces corresponds to a singular point on the other, thus it is not surprising that these notions appear together in Definition 3.1 .

In certain special cases, we can distinguish the singular points from the parabolic or flat points, which we will see here.

As another approach for considering singularities on discrete surfaces, motivated by the second author's work [32], we can define singular faces. We come back to this in Definition 8.1, and examine criteria for singular faces, and also their relationships with singular vertices in some special cases. 


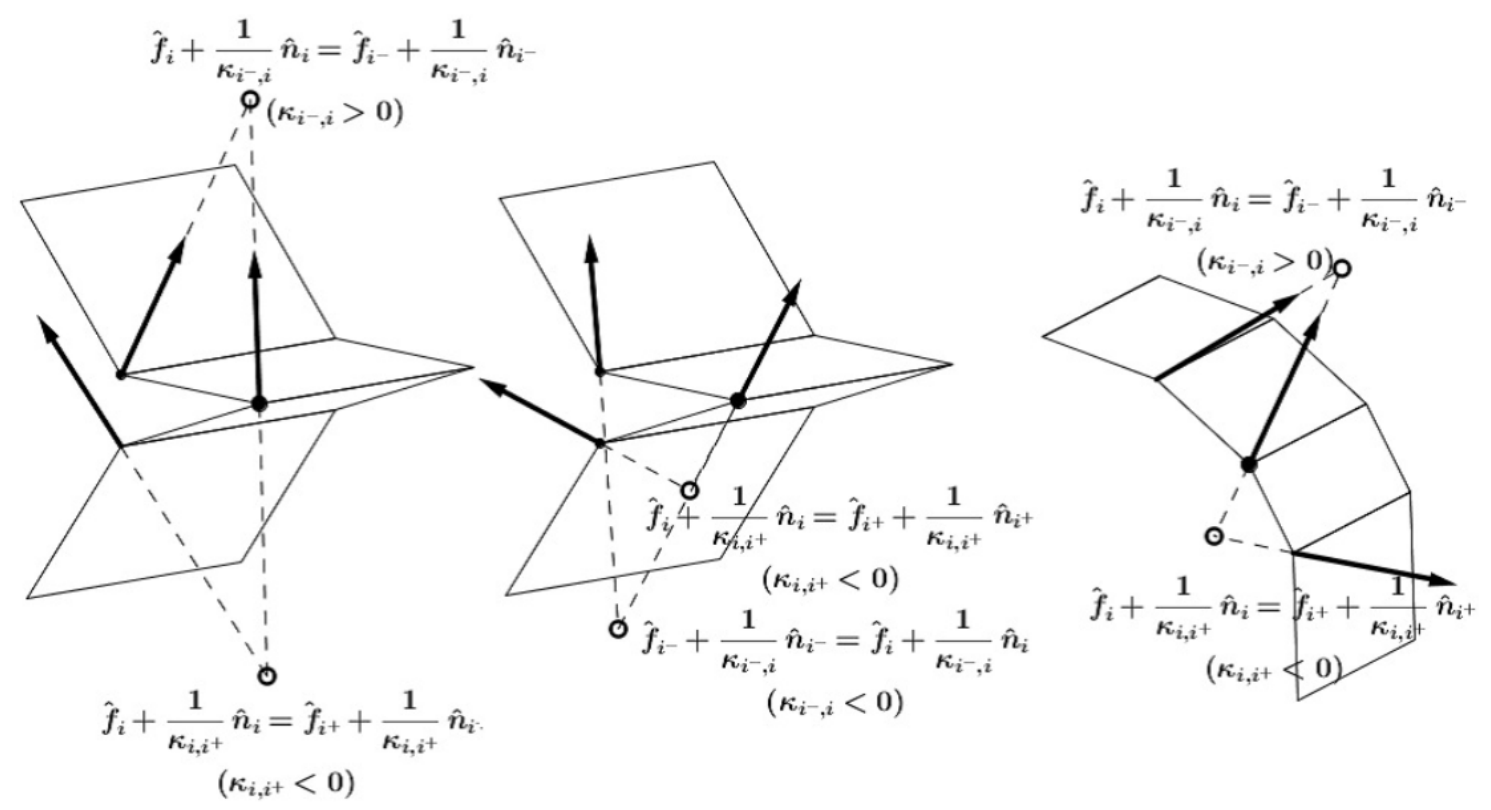

Figure 1. Examples of FPS vertices on the left and right, and a nonexample in the middle. The figure on the left shows a situation we should regard as a singularity, and the figure on the right shows what should be regarded as a flat or parabolic point. The figure in the middle is neither. Here, if $i=(m, n)$, then we have either $i^{+}=(m+1, n), i^{-}=(m-1, n)$ or $i^{+}=(m, n+1), i^{-}=(m, n-1)$.

\section{Smooth linear Weingarten surfaces of Bryant and Bianchi type in $\mathbb{H}^{3}$ AND $\mathbb{S}^{2,1}$}

We include this section to motivate the discretizations in $\$ 5$. In $\mathbb{R}^{3,1}$ with signature $(-,+,+,+)$, with points $\left(x_{0}, x_{1}, x_{2}, x_{3}\right) \in \mathbb{R}^{3,1}$ described in matrix form as

$$
X=\left(\begin{array}{cc}
x_{0}+x_{3} & x_{1}-i x_{2} \\
x_{1}+i x_{2} & x_{0}-x_{3}
\end{array}\right)
$$

the metric is $\langle X, Y\rangle=\frac{-1}{2} \operatorname{tr}\left(X\left(\begin{array}{cc}0 & -i \\ i & 0\end{array}\right) Y^{t}\left(\begin{array}{cc}0 & -i \\ i & 0\end{array}\right)\right)$. We define

$$
\begin{aligned}
& \mathbb{H}^{3}:=\left\{X \in \mathbb{R}^{3,1} \mid\langle X, X\rangle=-1\right\}=\left\{ \pm F \bar{F}^{t} \mid F \in \mathrm{SL}_{2} \mathbb{C}\right\} \\
& \mathbb{S}^{2,1}:=\left\{X \in \mathbb{R}^{3,1} \mid\langle X, X\rangle=1\right\}=\left\{F\left(\begin{array}{cc}
1 & 0 \\
0 & -1
\end{array}\right) \bar{F}^{t} \mid F \in \mathrm{SL}_{2} \mathbb{C}\right\} .
\end{aligned}
$$

We call a surface $\hat{f}$ in $\mathbb{H}^{3}$ a linear Weingarten surface of Bryant type (BrLW surface, for short) if $\hat{f}$ satisfies

$$
2 t\left(H_{\hat{f}}-1\right)+(1-t)\left(K_{e x t, \hat{f}}-1\right)=0,
$$

where $K_{\text {ext, } \hat{f}}$ and $H_{\hat{f}}$ are the extrinsic Gaussian and mean curvatures of $\hat{f}$ with respect to $\mathbb{H}^{3}$, and we call a surface $\hat{n}$ in $\mathbb{S}^{2,1}$ a linear Weingarten surface of Bianchi type (BiLW 
surface, for short) if $\hat{n}$ satisfies

$$
2 t\left(H_{\hat{n}}-1\right)-(1+t)\left(K_{\text {ext }, \hat{n}}-1\right)=0 .
$$

Solving

$$
d E=E\left(\begin{array}{cc}
0 & g^{\prime} \\
\left(g^{\prime}\right)^{-1} & 0
\end{array}\right) d z
$$

for $E \in \mathrm{SL}_{2}(\mathbb{C})$, where $g$ is a holomorphic function with nonzero derivative $g^{\prime}=\partial_{z} g$ on a Riemann surface $M^{2}$ with local coordinate $z$, we take, for any constant $t \in \mathbb{R}$,

$$
\left\{\begin{array}{r}
L=\left(\begin{array}{cc}
0 & \sqrt{\mathcal{T}} \\
\frac{-1}{\sqrt{\mathcal{T}}} & \frac{-t \bar{g}}{\sqrt{\mathcal{T}}}
\end{array}\right) \quad(\mathcal{T}:=1+t g \bar{g}), \\
\hat{f}=\operatorname{sgn}(\mathcal{T}) E L \overline{E L}^{t}, \quad \hat{n}=\operatorname{sgn}(\mathcal{T}) E L\left(\begin{array}{cc}
1 & 0 \\
0 & -1
\end{array}\right) \overline{E L}^{t},
\end{array}\right.
$$

making the genericity assumption $\mathcal{T} \neq 0$.

Then $\hat{f}$ is a BrLW surface in $\mathbb{H}^{3}$ with unit normal vector field $\hat{n}$, since $\langle\hat{f}, \hat{n}\rangle=$ $\langle d \hat{f}, \hat{n}\rangle=0$. Moreover, $\hat{n}$ is a BiLW surface in $\mathbb{S}^{2,1}$. Here we outline a proof of this.

The three fundamental forms of $\hat{f}$ become, with $h:=\left|g^{\prime}\right|^{-2} \mathcal{T}^{-2}$,

$$
\begin{aligned}
& I=h\left\{\left((1-t)\left|g^{\prime}\right|^{2}+\mathcal{T}^{2}\right)^{2} d x^{2}+\left((1-t)\left|g^{\prime}\right|^{2}-\mathcal{T}^{2}\right)^{2} d y^{2}\right\}, \\
& I I=-h\left\{\left(\left|g^{\prime}\right|^{4}-\left(t\left|g^{\prime}\right|^{2}-\mathcal{T}^{2}\right)^{2}\right) d x^{2}+\left(\left|g^{\prime}\right|^{4}-\left(t\left|g^{\prime}\right|^{2}+\mathcal{T}^{2}\right)^{2}\right) d y^{2}\right\}, \\
& I I I=h\left\{\left((1+t)\left|g^{\prime}\right|^{2}-\mathcal{T}^{2}\right)^{2} d x^{2}+\left((1+t)\left|g^{\prime}\right|^{2}+\mathcal{T}^{2}\right)^{2} d y^{2}\right\} .
\end{aligned}
$$

The principal curvatures of $\hat{f}$ and $\hat{n}$ are then

$$
\begin{aligned}
& k_{1, \hat{f}}=-\frac{(1+t)\left|g^{\prime}\right|^{2}-\mathcal{T}^{2}}{(1-t)\left|g^{\prime}\right|^{2}+\mathcal{T}^{2}}, \quad k_{2, \hat{f}}=-\frac{(1+t)\left|g^{\prime}\right|^{2}+\mathcal{T}^{2}}{(1-t)\left|g^{\prime}\right|^{2}-\mathcal{T}^{2}}, \\
& k_{1, \hat{n}}=\frac{1}{k_{1, \hat{f}}}, \quad k_{2, \hat{n}}=\frac{1}{k_{2, \hat{f}}}, \quad H_{\hat{f}}=\frac{H_{\hat{n}}}{K_{\text {ext }, \hat{n}}}, \quad K_{\text {ext }, \hat{f}}=\frac{1}{K_{\text {ext }, \hat{n}}},
\end{aligned}
$$

and so $\hat{f}$ satisfies Equation (3) and $\hat{n}$ satisfies Equation (4). In fact, all BrLW and BiLW surfaces without umbilics ( $g^{\prime}$ would be zero at umbilics) can be constructed this way, using holomorphic functions $g$.

Thus sufficient conditions for $\hat{f}$ and $\hat{n}$, respectively, to have singularities are

$$
\mathcal{T}^{4}=(1-t)^{2}\left|g^{\prime}\right|^{4}, \quad \mathcal{T}^{4}=(1+t)^{2}\left|g^{\prime}\right|^{4},
$$

respectively. For certain special values of $t$ these conditions simplify as follows:

$$
\begin{array}{llrl}
\hat{f} \text { with } t=0: & & \left|g^{\prime}\right|=1, \\
\hat{n} \text { with } t=0: & & \left|g^{\prime}\right|=1, \\
\hat{f} \text { with } t=1: & & \text { null condition, } \\
\hat{n} \text { with } t=-1: & & |g|=1 .
\end{array}
$$

Because $\hat{f}$ and $\hat{n}$ are smooth well-defined maps that can have singularities, it is natural to lift to Lie sphere geometry in $\mathbb{R}^{4,2}$, with

$$
f=(\hat{f}, 1,0)^{t}, \quad n=(\hat{n}, 0,1)^{t}
$$


determined by

$$
p=(0,0,0,0,0,1)^{t}, \quad q=(0,0,0,0,-1,0)^{t} .
$$

For a BrLW surface $\hat{f} \in \mathbb{H}^{3}=M_{p, q}^{3}$ with BiLW normal $\hat{n} \in \mathbb{S}^{2,1}=M_{q, p}^{3}$, we can define the Legendre lift $\Lambda=\operatorname{span}\left\{s_{+}, s_{-}\right\}$for

$$
s_{ \pm}=b_{ \pm} f+n \quad \text { with } \quad b_{+}=1 \text { and } b_{-}=\frac{t+1}{t-1},
$$

and then $s_{ \pm}$have constant conserved quantities

$$
q_{+}=(0,0,0,0,1,1)^{t}, \quad q_{-}=(0,0,0,0, t-1, t+1)^{t} .
$$

in the sense that $\left(s_{ \pm}, q_{ \pm}\right)=0$, equivalently the equations $\Gamma^{ \pm} q_{ \pm}=0$ for the associated families of flat connections hold (see [8], [26]). Furthermore, because $b_{ \pm}$are constant and because the elements $g_{i j}$ of the first fundamental form of $\hat{f}$ satisfy (using Equation (3))

$$
\pm \frac{\sqrt{g_{11}}}{\sqrt{g_{22}}}=\frac{1-\kappa_{2}}{1-\kappa_{1}}=\frac{-t-1+(t-1) \kappa_{2}}{t+1-(t-1) \kappa_{1}}
$$

all of Equations (4.5) and (4.10) and (4.11) in [26] hold, and so $s_{ \pm}$are isothermic sphere congruences. Thus $\Lambda$ is an $\Omega$ surface with a pair of constant conserved quantities.

Conversely, if we start with an $\Omega$ surface with constant conserved quantities $q_{ \pm}$for isothermic sphere congruences $s_{ \pm}=b_{ \pm} f+n$ respectively, we can reverse the above arguments to see that we obtain a BrLW surface $\hat{f}$ with BiLW normal $\hat{n}$ in the spaceforms $M_{p, q}^{3}$ and $M_{q, p}^{3}$, with $p$ and $q$ as above.

This proves the next lemma, which was already understood in [8]:

Lemma 4.1 ([8]). All smooth BrLW and BiLW surfaces in $\mathbb{H}^{3}$ and $\mathbb{S}^{2,1}$ are projections of $\Omega$ surfaces with constant conserved quantities, at least one of which is lightlike. Conversely, for any smooth $\Omega$ surface with constant conserved quantitie $\Psi_{ \pm}$, at least one of which is lightlike, its projections $\hat{f}$ and $\hat{n}$ given by choosing $p, q \in \operatorname{span}\left\{q_{ \pm}\right\}$are $B r L W$ and BiLW surfaces, respectively.

The same result holds for general linear Weingarten $\hat{f}$ and $\hat{n}$, even without the condition that at least one of the $q_{ \pm}$is lightlike, again see [8]. However, here we consider only the cases given in Lemma 4.1 .

\section{Discrete surfaces With Weierstrass-type Representations}

First we give Weierstrass-type representations for discrete surfaces using the more symmetric form of the base equation as in $\S 6$ of [19].

Let $g: \mathbb{Z}^{2} \rightarrow \mathbb{C}$ be a function satisfying

$$
\operatorname{cr}\left(g_{i}, g_{j}, g_{k}, g_{\ell}\right):=\frac{\left(g_{i}-g_{j}\right)\left(g_{k}-g_{\ell}\right)}{\left(g_{j}-g_{k}\right)\left(g_{\ell}-g_{i}\right)}=\frac{\alpha_{i j}}{\alpha_{i \ell}}<0
$$

where $\alpha_{i j}\left(\right.$ resp. $\left.\alpha_{i \ell}\right)$ is a scalar function defined on the horizontal edges (resp. vertical edges) and unchanging with respect to vertical (resp. horizontal) shifts. A complexvalued function $g$ satisfying the above condition is called a discrete holomorphic function and $\alpha_{i j}, \alpha_{i \ell}$ are called cross ratio factorizing functions. Now we assume the discrete

\footnotetext{
${ }^{1}$ We assume $q_{+}, q_{-}$are not parallel, and that $\operatorname{span}\left\{q_{ \pm}\right\}$is not a null plane.
} 
analog of $g^{\prime} \neq 0$, i.e. $d g_{i j}:=g_{j}-g_{i} \neq 0$ and $d g_{i \ell} \neq 0$ for all quadrilaterals. We again make the genericity assumption

$$
\mathcal{T}_{i}:=1+t g_{i} \overline{g_{i}} \neq 0
$$

for all vertices $i$, for the chosen constant $t \in \mathbb{R}$. Take $\lambda \in \mathbb{R}$ to be any non-zero constant so that $1-\lambda \alpha_{i j} \neq 0$ on all edges. Solving

$$
E_{i}^{-1} E_{j}=\frac{1}{\sqrt{1-\lambda \alpha_{i j}}}\left(\begin{array}{cc}
1 & d g_{i j} \\
\frac{\lambda \alpha_{i j}}{d g_{i j}} & 1
\end{array}\right)
$$

and the analogous equation with $j$ replaced by $\ell$, for $E_{i} \in \mathrm{SL}_{2} \mathbb{C}$ for all $i$, and defining

$$
L_{i}=\left(\begin{array}{cc}
0 & \sqrt{\mathcal{T}_{i}} \\
\frac{-1}{\sqrt{\mathcal{T}_{i}}} & \frac{-t \bar{g}_{i}}{\sqrt{\mathcal{T}_{i}}}
\end{array}\right),
$$

the surface $\hat{f}$ and its normal $\hat{n}$

$$
\hat{f}_{i}=\operatorname{sgn}\left(\mathcal{T}_{i}\right) E_{i} L_{i}{\overline{E_{i} L_{i}}}^{t}, \quad \hat{n}_{i}=\operatorname{sgn}\left(\mathcal{T}_{\mathrm{i}}\right) E_{i} L_{i}\left(\begin{array}{cc}
1 & 0 \\
0 & -1
\end{array}\right){\overline{E_{i} L_{i}}}^{t},
$$

we will see that these are discrete BrLW surfaces and BiLW surfaces in $\mathbb{H}^{3}$ and $\mathbb{S}^{2,1}$, respectively. Direct computations confirm the following lemma:

Lemma 5.1. For any choice of $t$, we have the following:

- $d \hat{f}_{i j}\left\|d \hat{n}_{i j}, d \hat{f}_{i \ell}\right\| d \hat{n}_{i \ell}$ in $\mathbb{R}^{3,1}$ for all edges $i j$, il, and the principal curvatures $\kappa_{i *}$ satisfy

$$
d n_{i *}=-\kappa_{i *} d f_{i *}
$$

and furthermore

$$
\kappa_{i *}=\frac{-\left|d g_{i *}\right|^{2}(1+t)+\left(1+t\left|g_{i}\right|^{2}\right)\left(1+t\left|g_{*}\right|^{2}\right) \lambda \alpha_{i *}}{-\left|d g_{i *}\right|^{2}(-1+t)+\left(1+t\left|g_{i}\right|^{2}\right)\left(1+t\left|g_{*}\right|^{2}\right) \lambda \alpha_{i *}},
$$

for $*=j, \ell$.

- $1+t\left|g_{i}\right|^{2}>0$, resp. $1+t\left|g_{i}\right|^{2}<0$, if and only if $\hat{f}_{i}$ lies in the upper, resp. lower, sheet of $\mathbb{H}^{3}$.

- $\hat{f}_{i}, \hat{f}_{j}, \hat{f}_{k}, \hat{f}_{\ell}$ lie in a plane in $\mathbb{R}^{3,1}$, and thus are concircular in $\mathbb{H}^{3}$.

Corollary 5.1. For any choice of $t$, the parallel surfaces

$$
\cosh \theta \cdot \hat{f}+\sinh \theta \cdot \hat{n}, \quad \cosh \theta \cdot \hat{n}+\sinh \theta \cdot \hat{f}
$$

are concircular for all $\theta \in \mathbb{R}$.

Proof. $d \hat{f}_{i *} \| d \hat{n}_{i *}$ and the fact that corresponding quadrilaterals of $f$ and $n$ lie in parallel planes imply that corresponding quadrilaterals of $\cosh \theta \cdot \hat{f}+\sinh \theta \cdot \hat{n}$ also lie in parallel planes, proving the corollary.

Like in Equation (6), we can lift $\hat{f}$ and $\hat{n}$ to $f, n \in \mathbb{R}^{4,2}$, producing a discrete Legendre immersion $\Lambda=\operatorname{span}\{f, n\}$. We define

$$
\mathcal{A}(f, f)_{i j k \ell}:=\frac{1}{2} d f_{i k} \wedge d f_{j \ell},
$$


and we can define real-valued functions $H=H_{\hat{f}}$ and $K=K_{\hat{f}}$ on faces by

$$
\begin{gathered}
\mathcal{A}(f, n)_{i j k \ell}:=\frac{1}{4}\left\{d f_{i k} \wedge d n_{j \ell}+d n_{i k} \wedge d f_{j \ell}\right\}=-H \cdot \mathcal{A}(f, f)_{i j k \ell}, \\
\mathcal{A}(n, n)_{i j k \ell}=\frac{1}{2} d n_{i k} \wedge d n_{j \ell}=K \cdot \mathcal{A}(f, f)_{i j k \ell} .
\end{gathered}
$$

We have the following definition:

Definition 5.1 ([9]). We call $K_{\hat{f}}$ and $H_{\hat{f}}$ the (extrinsic) Gaussian and mean curvature of the projection $\hat{f}$ of $\Lambda$ to the spaceform given by $p, q$.

Proven similarly to the corresponding result for $\mathbb{R}^{3}$ in [4], using item 1 of Lemma 5.1 . we have:

Lemma 5.2. For all choices of spaceforms, we have

$$
\begin{aligned}
& H_{\hat{f}}=\frac{\kappa_{i j} \kappa_{k \ell}-\kappa_{i \ell} \kappa_{j k}}{\kappa_{i j}-\kappa_{i \ell}-\kappa_{j k}+\kappa_{k \ell}}, \\
& K_{\hat{f}}=\frac{\kappa_{i j} \kappa_{j k} \kappa_{k \ell} \kappa_{i \ell}}{\kappa_{i j}-\kappa_{i \ell}-\kappa_{j k}+\kappa_{k \ell}}\left(-\frac{1}{\kappa_{i j}}+\frac{1}{\kappa_{j k}}+\frac{1}{\kappa_{i \ell}}-\frac{1}{\kappa_{k \ell}}\right) .
\end{aligned}
$$

Proof. The compatibility condition $\hat{n}_{i j}+\hat{n}_{j k}=\hat{n}_{i \ell}+\hat{n}_{\ell k}$ for $\hat{n}$ implies

$$
\kappa_{i j} d \hat{f}_{i j}+\kappa_{j k} d \hat{f}_{j k}=\kappa_{i \ell} d \hat{f}_{i \ell}+\kappa_{k \ell} d \hat{f}_{\ell k} .
$$

Thus

$$
\begin{aligned}
& \kappa_{i j} d \hat{f}_{i j}+\kappa_{j k}\left(d \hat{f}_{i k}-d \hat{f}_{i j}\right)=\kappa_{i \ell} d \hat{f}_{i \ell}+\kappa_{k \ell}\left(d \hat{f}_{i k}-d \hat{f}_{i \ell}\right) \\
& \Rightarrow d \hat{f}_{i k}=c_{1} d \hat{f}_{i j}+c_{2} d \hat{f}_{i \ell}
\end{aligned}
$$

where

$$
c_{1}=\frac{-\kappa_{i j}+\kappa_{j k}}{\kappa_{j k}-\kappa_{k \ell}}, \quad c_{2}=\frac{-\kappa_{l k}+\kappa_{i \ell}}{\kappa_{j k}-\kappa_{k \ell}} .
$$

Similarly, by the compatibility condition for $\hat{f}$ and the condition $d n_{* *}=-\kappa_{* *} d f_{* *}$, we have $d \hat{n}_{i k}=c_{3} d f_{i j}+c_{4} d f_{i l}$, where

$$
c_{3}=\frac{\kappa_{k \ell}\left(\kappa_{j k}-\kappa_{i j}\right)}{\kappa_{k \ell}-\kappa_{j k}}, \quad c_{4}=\frac{\kappa_{j k}\left(\kappa_{i \ell}-\kappa_{k \ell}\right)}{\kappa_{k \ell}-\kappa_{j k}} .
$$

Note that $d \hat{f}_{j \ell}=d \hat{f}_{i \ell}-d \hat{f}_{i j}, d \hat{n}_{j \ell}=d \hat{n}_{i \ell}-d \hat{n}_{i j}$, and we have

$$
H_{\hat{f}}=\frac{\kappa_{i \ell} c_{1}+\kappa_{i j} c_{2}-c_{3}-c_{4}}{2\left(c_{1}+c_{2}\right)}, K_{\hat{f}}=-\frac{\kappa_{i \ell} c_{3}+\kappa_{i j} c_{4}}{c_{1}+c_{2}} .
$$

Substituting Equations (10) and (11) into Equation (12), we have $H_{\hat{f}}$ and $K_{\hat{f}}$ as in Lemma 5.2.

We can similarly define the Gaussian and mean curvatures $K_{\hat{n}}, H_{\hat{n}}$ of $\hat{n}$, and we see that

$$
K_{\hat{n}}=\frac{1}{K_{\hat{f}}}, \quad H_{\hat{n}}=\frac{H_{\hat{f}}}{K_{\hat{f}}}, \quad \kappa_{i j, \hat{n}}=\frac{1}{\kappa_{i j, \hat{f}}}, \quad \kappa_{i l, \hat{n}}=\frac{1}{\kappa_{i l, \hat{f}}} .
$$

One can confirm the next lemma via Lemma 5.2 and Equations (7), (13): 

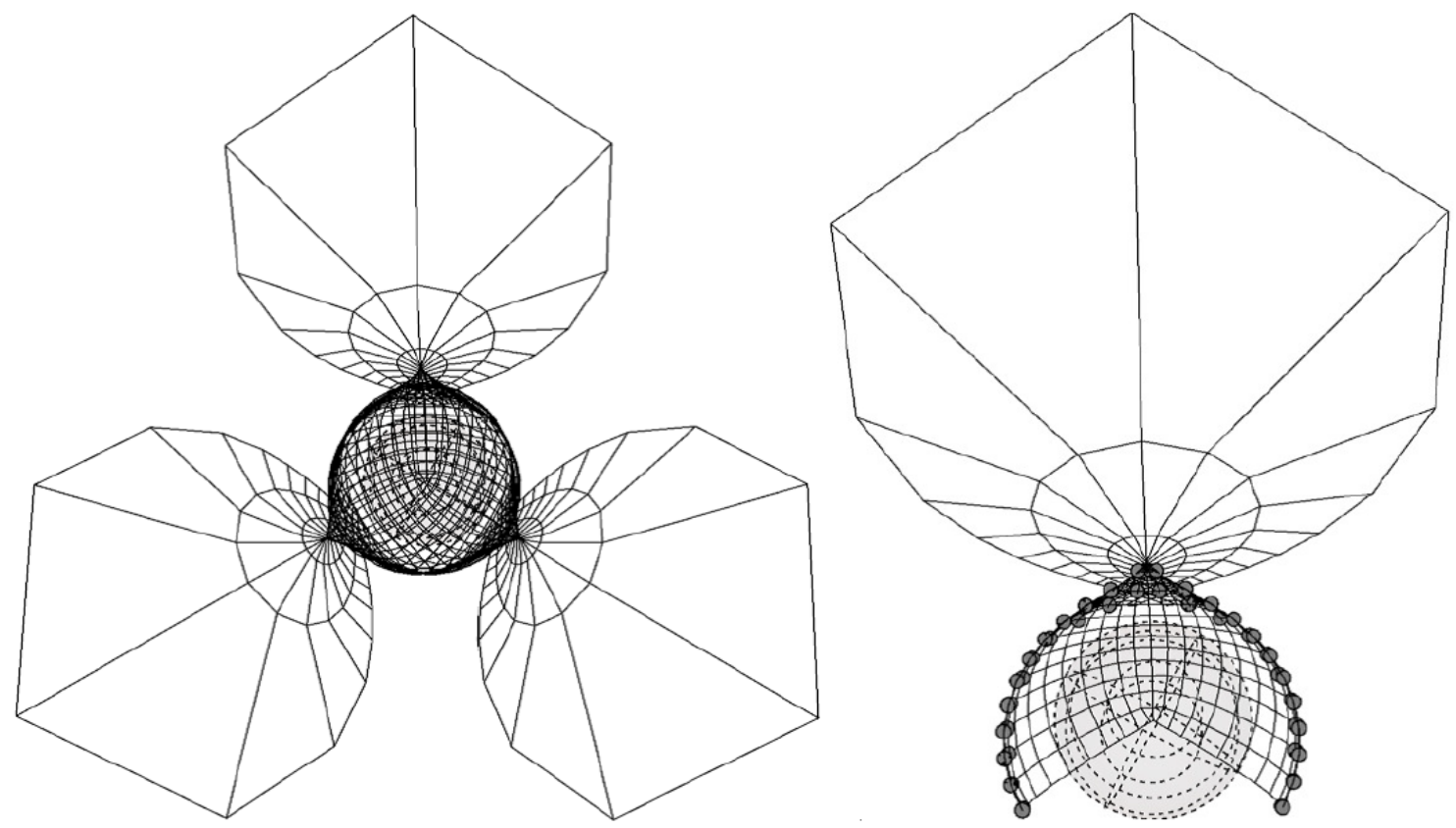

Figure 2. One example of a discrete BrLW surface in $\mathbb{H}^{3}$ with $t=-2$. Red vertices are the FPS vertices of the surface. In order to draw the surface, we use stereographic projection from the south pole $(0,0,0,-1)$. When $1+t\left|g_{i}\right|^{2}>0$, the surface is projected to the inside of the unit ball $\mathbb{B}^{3}$, whose boundary is drawn in gray above. When $1+t\left|g_{i}\right|^{2}<0$, it is projected to the outside of $\mathbb{B}^{3}$. One-third of the surface is shown on the right.

Lemma 5.3. The mean and Gaussian curvatures $H_{\hat{f}}$ and $K_{\hat{f}}$ of a discrete surface $\hat{f}$ with Weierstrass-type representation in $\mathbb{H}^{3}$ satisfy

$$
2 t\left(H_{\hat{f}}-1\right)+(1-t)\left(K_{\hat{f}}-1\right)=0,
$$

and the mean and Gaussian curvatures $H_{\hat{n}}$ and $K_{\hat{n}}$ of a discrete surface $\hat{n}$ with Weierstrasstype representation in $\mathbb{S}^{2,1}$ satisfy

$$
2 t\left(H_{\hat{n}}-1\right)-(1+t)\left(K_{\hat{n}}-1\right)=0 .
$$

Thus we know that the discrete surfaces with Weierstrass-type representations defined here are included amongst the discrete BrLW and BiLW surfaces defined in [9], by the following Proposition 5.1 from [9]. This proposition also includes discrete minimal surfaces in $\mathbb{R}^{3}$ and their parallel surfaces in $\mathbb{R}^{3}$, as well as parallel surfaces of discrete maximal surfaces in $\mathbb{R}^{2,1}$.

Proposition 5.1 ([9]). All discrete BrLW and BiLW surfaces in $\mathbb{H}^{3}$ and $\mathbb{S}^{2,1}$, and all parallel surfaces of discrete minimal surfaces in $\mathbb{R}^{3}$ and discrete maximal surfaces in $\mathbb{R}^{2,1}$, are projections of discrete $\Omega$ surfaces with constant conserved quantities, at least one of which is lightlike. Conversely, for any discrete $\Omega$ surface with constant conserved 
quantitie $2^{2} q_{ \pm}$, at least one of which is lightlike, its projections $\hat{f}$ and $\hat{n}$ given by choosing $p, q \in \operatorname{span}\left\{q_{ \pm}\right\}$are discrete BrLW and BiLW surfaces, respectively, or $\hat{f}$ is either a parallel surface of a discrete minimal surface in $\mathbb{R}^{3}$ or maximal surface in $\mathbb{R}^{2,1}$.

In the smooth case, as mentioned in [23], parallel surfaces of BrLW surfaces in $\mathbb{H}^{3}$ are also BrLW surfaces, and BrLW surfaces are classified into the following three types:

(1) flat surfaces (BrLW surfaces with $t=0)$,

(2) linear Weingarten surfaces of hyperbolic type (BrLW surfaces with $t>0$ ),

(3) linear Weingarten surfaces of de Sitter type (BrLW surfaces with $t<0$ ).

Parallel surfaces of each type belong to the same type. Thus parallel surfaces of a flat front are also flat. Likewise, parallel surfaces of the other two types again belong to the same types.

Here we see that the same result as in the smooth case holds also in the discrete case. Let $\hat{f}$ be a discrete BrLW surface in $\mathbb{H}^{3}$. From [24, we have that the Gaussian and mean curvatures $K_{\hat{f}}^{\theta}, H_{\hat{f}}^{\theta}$ of the parallel surface $\hat{f}^{\theta}$ at oriented distance $\theta$ are

$$
\begin{aligned}
K_{\hat{f}}^{\theta} & =\frac{K_{\hat{f}} \cosh ^{2} \theta-H_{\hat{f}} \sinh 2 \theta+\sinh ^{2} \theta}{\cosh ^{2} \theta-H_{\hat{f}} \sinh 2 \theta+K_{\hat{f}} \sinh ^{2} \theta}, \\
H_{\hat{f}}^{\theta} & =-\frac{\left(K_{\hat{f}}+1\right) \sinh (2 \theta)-2 H_{\hat{f}} \cosh (2 \theta)}{2\left\{\cosh ^{2} \theta-H_{\hat{f}} \sinh (2 \theta)+K_{\hat{f}} \sinh ^{2} \theta\right\}} .
\end{aligned}
$$

Observing that $\hat{f}=\left(\hat{f}^{\theta}\right)^{-\theta}$, we have

$$
\left\{\begin{array}{c}
K_{\hat{f}}=\frac{K_{\hat{f}}^{\theta} \cosh ^{2} \theta+H_{\hat{f}}^{\theta} \sinh 2 \theta+\sinh ^{2} \theta}{\cosh ^{2} \theta+H_{\hat{f}}^{\theta} \sinh 2 \theta+K_{\hat{f}}^{\theta} \sinh ^{2} \theta}, \\
H_{\hat{f}}=\frac{\left(K_{\hat{f}}^{\theta}+1\right) \sinh (2 \theta)+2 H_{\hat{f}}^{\theta} \cosh (2 \theta)}{2\left\{\cosh ^{2} \theta+H_{\hat{f}}^{\theta} \sinh (2 \theta)+K_{\hat{f}}^{\theta} \sinh ^{2} \theta\right\}} .
\end{array}\right.
$$

Substituting Equation (16) into Equation (14), we have

$$
2 T\left(H_{\hat{f}}^{\theta}-1\right)+(1-T)\left(K_{\hat{f}}^{\theta}-1\right)=0,
$$

where $T=\mathrm{e}^{-2 \theta} t$. Thus discrete BrLW surfaces in $\mathbb{H}^{3}$ are classified into the three types $(1)-(3)$ mentioned above. Similarly, discrete BiLW surfaces in $\mathbb{S}^{2,1}$ are classified into three types.

\section{Singular vertices on discrete nonzero CGC Surfaces in $M^{3}$}

When a smooth surface has CGC $K=\kappa_{1} \kappa_{2} \neq 0$, then when one of the $\kappa_{\alpha}$ passes through zero, the other passes through infinity, and we can always call this a singular point. This is precisely what allowed for the description of singularities of discrete flat (i.e. $K \equiv 1$ ) surfaces in $\mathbb{H}^{3}$ as given in [19]. Here we develop that into a definition without reliance on a Weierstrass type representation, extending it to all discrete surfaces in any $M^{3}$ with nonzero constant Gaussian curvature.

\footnotetext{
${ }^{2}$ Again we assume $q_{+}, q_{-}$are not parallel, and that $\operatorname{span}\left\{q_{ \pm}\right\}$is not a null plane.
} 
Definition 6.1. Consider $\Lambda$ as in Definition 2.1, together with a choice of spaceform determined by choosing $p$ and $q$, that has projection $\hat{f}$ with nonzero constant discrete Gaussian curvature $K_{\hat{f}}$. We say that $(m, n)$ is a singular vertex of $\hat{f}$ if

$$
\kappa_{(m-1, n),(m, n)} \cdot \kappa_{(m, n),(m+1, n)} \leq 0 \quad \text { or } \quad \kappa_{(m, n-1),(m, n)} \cdot \kappa_{(m, n),(m, n+1)} \leq 0 .
$$

For a $K \equiv 1$ surface with Weierstrass-type representation in $\mathbb{H}^{3}$, it was shown in [19] that, without loss of generality, $\left|\kappa_{(m, n),(m, n+1)}\right|>1$ and $\left|\kappa_{(m, n),(m+1, n)}\right|<1$ for all $m$ and $n$, which we note in the following theorem:

Theorem 6.1. In the case of a $K \equiv 1$ surface in $\mathbb{H}^{3}$ with Weierstrass-type representation so that the horizontal edges have principal curvatures with absolute value greater than 1, the first inequality in Definition 6.1 is equivalent to the definition of singular vertices for discrete flat surfaces in $\mathbb{H}^{3}$ as given in [19].

Proof. By Lemma 5.1, for $t=0$ we have

$$
\kappa_{i *}=\frac{-\left|d g_{i *}\right|^{2}+\lambda \alpha_{i *}}{\left|d g_{i *}\right|^{2}+\lambda \alpha_{i *}} .
$$

Let $p_{-}, p$ and $p_{+}$be three consecutive vertices in one direction in the lattice domain. We can define singularities on discrete flat (i.e. $K \equiv 1$ ) surfaces in $\mathbb{H}^{3}$, now without referring to caustics as in [19], by simply using the condition

$$
\frac{-\left|d g_{p_{-}}\right|^{2}+\lambda \alpha_{p_{-}}}{\left|d g_{p_{-} p}\right|^{2}+\lambda \alpha_{p_{-} p}} \cdot \frac{-\left|d g_{p p_{+}}\right|^{2}+\lambda \alpha_{p p_{+}}}{\left|d g_{p p_{+}}\right|^{2}+\lambda \alpha_{p p_{+}}}<0,
$$

as understood in [19].

However, our definition allows the second inequality in Definition 6.1, which allows us to include more singular vertices (see Figure 3).

\section{Discrete minimal SURFACES AND their PARAllel SURFACES}

7.1. Smooth minimal surfaces in $\mathbb{R}^{3}$. We can always take a smooth constant mean curvature (CMC) surface in a 3-dimensional Riemannian spaceform to have local isothermic coordinates $z=u+i v$ on $M^{2}, u, v \in \mathbb{R}$ (away from umbilic points), and then the Hopf differential becomes $r d z^{2}$ for some real constant $r$. Rescaling the coordinate $z$ by a constant real factor, we may assume $r=1$. So we now assume we have an isothermic minimal surface in $\mathbb{R}^{3}$ with Hopf differential $Q=d z^{2}$. Then

$$
\frac{Q}{d g}=\frac{d z}{g^{\prime}}
$$

where $g$ is the stereographic projection of the Gauss map to the complex plane, and $g^{\prime}=d g / d z$. The map $g$ taking $z$ in the domain of the immersion (of the surface) to $\mathbb{C}$ is holomorphic. We avoid umbilics, so we have $g^{\prime} \neq 0$. We are concerned with only local behavior, so we can ignore the possibility that $g$ has poles. Then the Weierstrass representation is

$$
\hat{f}=\operatorname{Re} \int_{z_{0}}^{z}\left(1-g^{2}, i+i g^{2}, 2 g\right)^{t} \frac{d z}{g^{\prime}}
$$




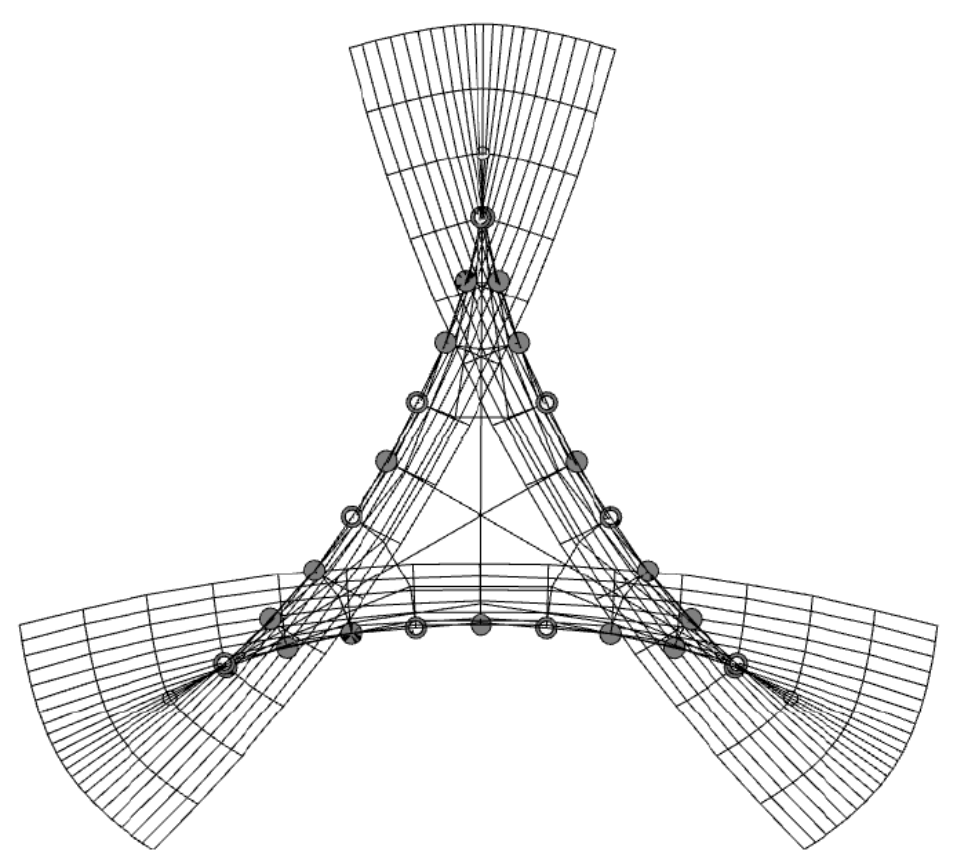

Figure 3. A flat surface in $\mathbb{H}^{3}$ with its singular vertices in the sense of [19] shown with solid gray dots, and the extra singular vertices that would be included by Definition 6.1 shown with hollowed-out dots.

with the last factor coming from (17). The metric of $\hat{f}$ is

$$
\frac{\left(1+|g|^{2}\right)^{2}}{\left|g^{\prime}\right|^{2}} d z d \bar{z} \text {. }
$$

By direct computation, we have:

Lemma 7.1. For a smooth minimal surface $\hat{f}$ as given in Equation (18), the partial derivatives of $\hat{f}$ are

$$
\begin{gathered}
\hat{f}_{u}=\operatorname{Re}\left(\frac{1-g^{2}}{g_{u}}, \frac{i\left(1+g^{2}\right)}{g_{u}}, \frac{2 g}{g_{u}}\right)^{t}, \\
\hat{f}_{v}=-\operatorname{Re}\left(\frac{1-g^{2}}{g_{v}}, \frac{i\left(1+g^{2}\right)}{g_{v}}, \frac{2 g}{g_{v}}\right)^{t} .
\end{gathered}
$$

Furthermore, the principal curvatures of the surface are

$$
\pm \kappa_{1}=\mp \kappa_{2}=\frac{2\left|g^{\prime}\right|^{2}}{\left(1+|g|^{2}\right)^{2}} .
$$

The next lemma will be used as motivation for the discussion about discrete minimal surfaces that follows it:

Lemma 7.2. Any parallel surface of a minimal surface in $\mathbb{R}^{3}$ without umbilics will have constant harmonic mean curvature, and will have neither parabolic nor flat points. 
Proof. For surfaces in $\mathbb{R}^{3}$ with Gaussian and mean curvatures $K$ and $H$, the parallel surfaces at distance $\rho$ have Gaussian and mean curvatures

$$
\hat{K}=\frac{K}{1-2 \rho H+\rho^{2} K}, \quad \hat{H}=\frac{H-\rho K}{1-2 \rho H+\rho^{2} K},
$$

so when we have a minimal surface $(H=0)$ in $\mathbb{R}^{3}$,

$$
\hat{K}=\frac{K}{1+\rho^{2} K}, \quad \hat{H}=\frac{-\rho K}{1+\rho^{2} K} .
$$

If the minimal surface has no umbilics, then $K \neq 0$, which implies no parallel surface can have any parabolic or flat points.

The parallel surfaces all have constant harmonic mean curvature since $\frac{\hat{H}}{\hat{K}}=-\rho$.

7.2. Discrete minimal surfaces in $\mathbb{R}^{3}$. Analogously to the smooth case, a suitable representation (or definition, see [3], [16]) for discrete minimal surfaces (equivalently, defined as discrete surfaces with $\left.H_{\hat{f}} \equiv 0\right)$ is, with $*=j, \ell$,

$$
\hat{f}_{*}-\hat{f}_{i}=\frac{\alpha_{i *}}{2} \operatorname{Re}\left(\frac{1-g_{*} g_{i}}{g_{*}-g_{i}}, \frac{\sqrt{-1}\left(1+g_{*} g_{i}\right)}{g_{*}-g_{i}}, \frac{g_{*}+g_{i}}{g_{*}-g_{i}}\right)^{t},
$$

where the map $g$ from a domain in $\mathbb{Z}^{2}$ to $\mathbb{C}$ is a discrete holomorphic function with cross ratio factorizing function $\alpha_{i *}$. As in the smooth case where we avoided umbilics, likewise here we assume

$$
g_{*}-g_{i} \neq 0 .
$$

Example 7.1. The discrete holomorphic function $g=c(m+n \sqrt{-1})$ for $c$ a complex constant will produce a discrete minimal Enneper surface. The discrete holomorphic function $g=e^{c_{1} m+c_{2} n \sqrt{-1}}$ for choices of real constants $c_{1}$ and $c_{2}$ so that the cross ratio is identically -1 will produce a discrete minimal catenoid. (See [3] for graphics.)

Furthermore, the principal curvatures $\kappa_{i *}$ defined on edges (similarly to (20)) are

$$
\kappa_{i *}=-\frac{4\left|d g_{i *}\right|^{2}}{\alpha_{i *}\left(1+\left|g_{i}\right|^{2}\right)\left(1+\left|g_{*}\right|^{2}\right)} .
$$

Based on Lemma 7.2, we can justify the following definition:

Definition 7.1. For any discrete minimal surface, we say that $(m, n)$ is a singular vertex of any given parallel surface if the principal curvatures $\kappa_{* *}$ of that parallel surface satisfy

$$
\begin{gathered}
\kappa_{(m-1, n),(m, n)} \cdot \kappa_{(m, n),(m+1, n)} \leq 0 \text { or } \\
\kappa_{(m, n-1),(m, n)} \cdot \kappa_{(m, n),(m, n+1)} \leq 0 .
\end{gathered}
$$

\section{Discrete maXimal SURFACES AND THeir PARALlel SURFACES}

Here we give the analogous situation as in $\$ 7$, but now in Lorentz 3-space. 

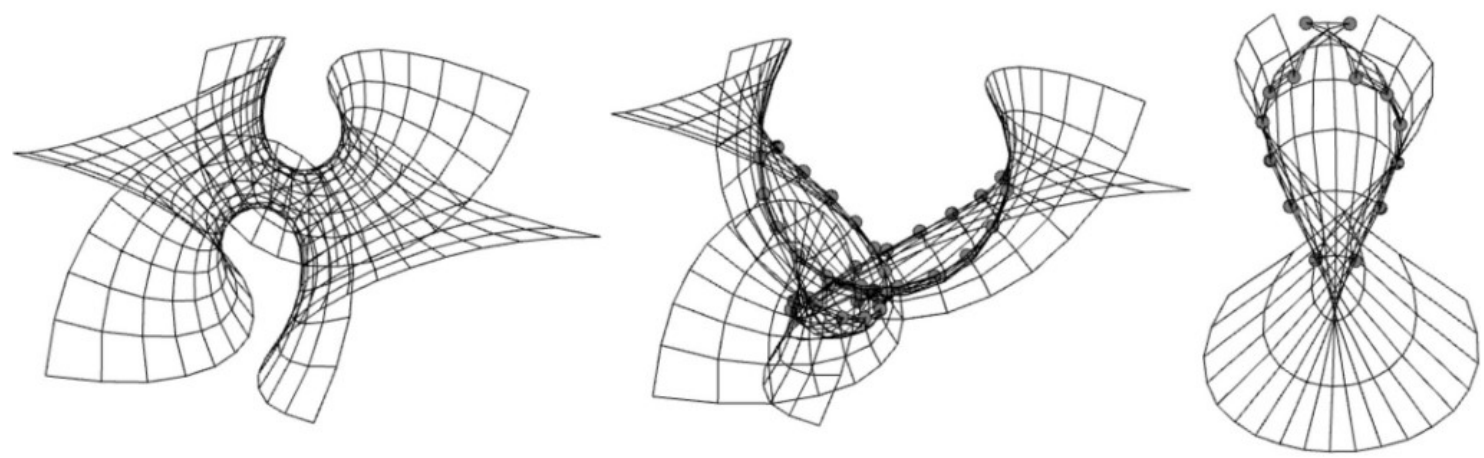

FiguRE 4. A discrete higher-order Enneper minimal surface in $\mathbb{R}^{3}$, its parallel surface at distance 20, and a one-third piece of the parallel surface, with singular vertices marked.

8.1. Smooth maximal surfaces in $\mathbb{R}^{2,1}$. First we briefly review smooth maximal surfaces. Let

$$
\mathbb{R}^{2,1}:=\left(\left\{\left(x_{1}, x_{2}, x_{0}\right)^{t} \mid x_{j} \in \mathbb{R}\right\},\langle\cdot, \cdot\rangle\right)
$$

be 3-dimensional Minkowski space with the Lorentz metric signature $(+,+,-)$.

Note that, for fixed $d \in \mathbb{R}$ and vector $n \in \mathbb{R}^{2,1} \backslash\{0\}$, a plane $\mathcal{P}=\left\{x \in \mathbb{R}^{2,1}\right.$ $\langle x, n\rangle=d\}$ is spacelike or timelike or lightlike when $n$ is timelike or spacelike or lightlike, respectively. Furthermore, a smooth surface in $\mathbb{R}^{2,1}$ is called spacelike if its tangent planes are spacelike. Let

$$
\hat{f}: M^{2} \rightarrow \mathbb{R}^{2,1}
$$

be a conformal immersion, where $M^{2}$ is a simply-connected domain in $\mathbb{C}$ with complex coordinate $z=u+i v(u, v \in \mathbb{R})$. $\hat{f}$ is a maximal surface if it is spacelike (which follows automatically from the conformality condition) with mean curvature identically 0 .

Defining

$$
\begin{aligned}
& \mathbb{H}_{+}^{2}:=\left\{x=\left(x_{1}, x_{2}, x_{0}\right)^{t} \in \mathbb{R}^{2,1} \mid\langle x, x\rangle=-1, x_{0}>0\right\}, \\
& \mathbb{H}_{-}^{2}:=\left\{x=\left(x_{1}, x_{2}, x_{0}\right)^{t} \in \mathbb{R}^{2,1} \mid\langle x, x\rangle=-1, x_{0}<0\right\},
\end{aligned}
$$

we have the following statement, analogous to the case of smooth minimal surfaces in $\mathbb{R}^{3}$, as in (18) (and having a similar proof): Away from umbilic points, smooth maximal surfaces lie in the class of isothermic surfaces, and each such surface can be represented with isothermic coordinates $(u, v), z=u+i v$, as

$$
\hat{f}=\operatorname{Re} \int\left(1+g^{2}, i\left(1-g^{2}\right),-2 g\right)^{t} \frac{d z}{g^{\prime}}
$$

for some choice of smooth holomorphic function $g: M^{2} \rightarrow \mathbb{C}$. The Gauss map of $\hat{f}$ lies in $\mathbb{H}_{+}^{2} \cup \mathbb{H}_{-}^{2}$, and its stereographic projection to $\mathbb{C}$ is $g$. 
Differentiating Equation (22) gives the following equations (analogous to Lemma 7.1):

$$
\begin{gathered}
\hat{f}_{u}=\operatorname{Re}\left(\frac{1+g^{2}}{g_{u}}, \frac{i\left(1-g^{2}\right)}{g_{u}},-\frac{2 g}{g_{u}}\right)^{t}, \\
\hat{f}_{v}=-\operatorname{Re}\left(\frac{1+g^{2}}{g_{v}}, \frac{i\left(1-g^{2}\right)}{g_{v}},-\frac{2 g}{g_{v}}\right)^{t} .
\end{gathered}
$$

Remark. Unlike the case of the Weierstrass representation for minimal surfaces in $\mathbb{R}^{3}$, smooth maximal surfaces in $\mathbb{R}^{2,1}$ have singularities when $|g|=1$, because the metrics

$$
\frac{\left(1-|g|^{2}\right)^{2}}{\left|g^{\prime}\right|^{2}} d z d \bar{z}
$$

of the smooth maximal surfaces can degenerate, due to the minus sign in the numerator in Equation (23), unlike the plus sign we have for the metrics of minimal surfaces in $\mathbb{R}^{3}$, as in 19 .

The principal curvatures of $\hat{f}$ are (analogous to $20 p$ )

$$
\pm \kappa_{1}=\mp \kappa_{2}=\frac{2\left|g^{\prime}\right|^{2}}{\left(1-|g|^{2}\right)^{2}} \text {. }
$$

By exactly the same proof as for Lemma 7.2 , we have:

Lemma 8.1. Any parallel surface of a maximal surface in $\mathbb{R}^{2,1}$ without umbilics will have constant harmonic mean curvature, and will have neither parabolic nor flat points.

8.2. Discrete maximal surfaces in $\mathbb{R}^{2,1}$. The following theorem was proven in 32 ] (analogous to (21)):

Proposition 8.1. Discrete maximal surfaces $\hat{f}$ (defined as discrete surfaces with $H_{\hat{f}} \equiv$ 0 in $\mathbb{R}^{2,1}$ ), maps from $\mathbb{Z}^{2}$ (or some subdomain) to $\mathbb{R}^{2,1}$, can be constructed using discrete holomorphic functions $g$ from the same domain to the complex plane $\mathbb{C}$ by solving

$$
\hat{f}_{*}-\hat{f}_{i}=\frac{\alpha_{i *}}{2} \operatorname{Re}\left(\frac{1+g_{*} g_{i}}{g_{*}-g_{i}}, \frac{\sqrt{-1}\left(1-g_{*} g_{i}\right)}{g_{*}-g_{i}},-\frac{g_{*}+g_{i}}{g_{*}-g_{i}}\right)^{t},
$$

with $\alpha_{i *}$ the cross ratio factorizing functions for $g$. Conversely, any discrete maximal surface satisfies (24) for some discrete holomorphic function $\mathrm{g}$.

Lemma 8.2. The principal curvatures $\kappa_{i *}$ of $\hat{f}$ defined on edges are

$$
\kappa_{i *}=-\frac{4\left|d g_{i *}\right|^{2}}{\alpha_{i *}\left(1-\left|g_{i}\right|^{2}\right)\left(1-\left|g_{*}\right|^{2}\right)} .
$$

We recall the following definition of singular faces as in [32]:

Definition 8.1. A face of $\hat{f}$ with vertices $\hat{f}_{i}, \hat{f}_{j}, \hat{f}_{k}, \hat{f}_{\ell}$ is singular if those four vertices lie in a non-spacelike plane.

It was proven in 32 that a quadrilateral of $\hat{f}$ is singular if and only if the corresponding circumcircle of $g$ intersects the unit circle $\mathbb{S}^{1} \subset \mathbb{C}$. From this we can conclude the following theorem: 
Theorem 8.1. Let $p_{-}, p$ and $p_{+}$be three consecutive vertices in one direction in the lattice domain of a maximal surface $\hat{f}$ in $\mathbb{R}^{2,1}$, with corresponding values $g_{-}, g$ and $g_{+}$for the discrete holomorphic function in the Weierstrass type representation (24). Suppose $p$ is an FPS vertex. Then the pair of faces adjacent to the edge $p_{-} p$ are singular, or the pair of faces adjacent to the edge $p_{+}$are singular, including the possibility that all four faces are singular.

Proof. Because

$$
\kappa_{p_{-} p} \kappa_{p p_{+}}=(\text {nonnegative term })\left(1-\left|g_{p_{-}}\right|^{2}\right)\left(1-\left|g_{p_{+}}\right|^{2}\right),
$$

$\kappa_{p_{-} p} \kappa_{p p_{+}}<0$ implies at least one of $\left(1-\left|g_{p}\right|^{2}\right)\left(1-\left|g_{p_{-}}\right|^{2}\right)$ or $\left(1-\left|g_{p}\right|^{2}\right)\left(1-\left|g_{p_{+}}\right|^{2}\right)$ is negative, and so at least one of the edges $p_{-} p$ or $p p_{+}$has two adjacent singular faces.

Theorem 8.1 indicates one reason why we should regard, in the case of discrete maximal surfaces, all FPS vertices as singular. In fact, like in the case of discrete minimal surfaces, Lemma 8.1 indicates we can say the same of parallel surfaces of discrete maximal surfaces as well:

Definition 8.2. For any discrete maximal surface, we say that $(m, n)$ is a singular vertex of any given parallel surface (allowing also for the initial maximal surface itself) if the principal curvatures of the parallel surface satisfy

$$
\begin{gathered}
\kappa_{(m-1, n),(m, n)} \cdot \kappa_{(m, n),(m+1, n)} \leq 0 \text { or } \\
\kappa_{(m, n-1),(m, n)} \cdot \kappa_{(m, n),(m, n+1)} \leq 0 .
\end{gathered}
$$

\section{Singular faces on discrete CMC 1 surfaces With Weierstrass-Type REPRESENTATIONS IN $\mathbb{S}^{2,1}$}

As in Definition 8.1, a quadrilateral of a discrete CMC 1 surface $\hat{n}$ with Weierstrasstype representation in $\mathbb{S}^{2,1}$ is singular if it does not lie in a spacelike plane. We give a geometric condition (Theorem 9.1) for when a quadrilateral of $\hat{n}$ is singular, analogous to a condition in the case of discrete maximal surfaces (see [32]). We then prove a relation (Corollary 9.1) between FPS vertices and singular faces on discrete CMC 1 faces in $\mathbb{S}^{2,1}$ (similar to Theorem 8.1), a relation that helps indicate which of the FPS vertices are actually singular.

The condition for a singular face to occur is

$$
\left(d \hat{f}_{i j}, d \hat{f}_{i j}\right)\left(d \hat{f}_{i \ell}, d \hat{f}_{i \ell}\right)-\left(d \hat{f}_{i j}, d \hat{f}_{i \ell}\right)^{2} \leq 0 .
$$

In the smooth CMC 1 case, with $g$ as in $\$ 4$, the singularities occur exactly where $|g|=$ 1. The condition is still $|g|=1$ even under the coordinate transformation $z \rightarrow \sqrt{\lambda \alpha} z$. The next proposition and theorem are the corresponding condition in the discrete case to $|g|=1$, and can be proven by computationally spelling out Equation 25. We define

$$
\begin{array}{r}
h_{1}=\left(1-\left|g_{j}\right|^{2}\right)\left|d g_{i \ell}\right|^{2}\left(1-\lambda \alpha_{i j}\right), \\
h_{2}=\left(1-\left|g_{\ell}\right|^{2}\right)\left|d g_{i j}\right|^{2}\left(1-\lambda \alpha_{i \ell}\right), \\
h_{3}=\left(1-\left|g_{i}\right|^{2}\right)\left|d g_{j \ell}\right|^{2} .
\end{array}
$$



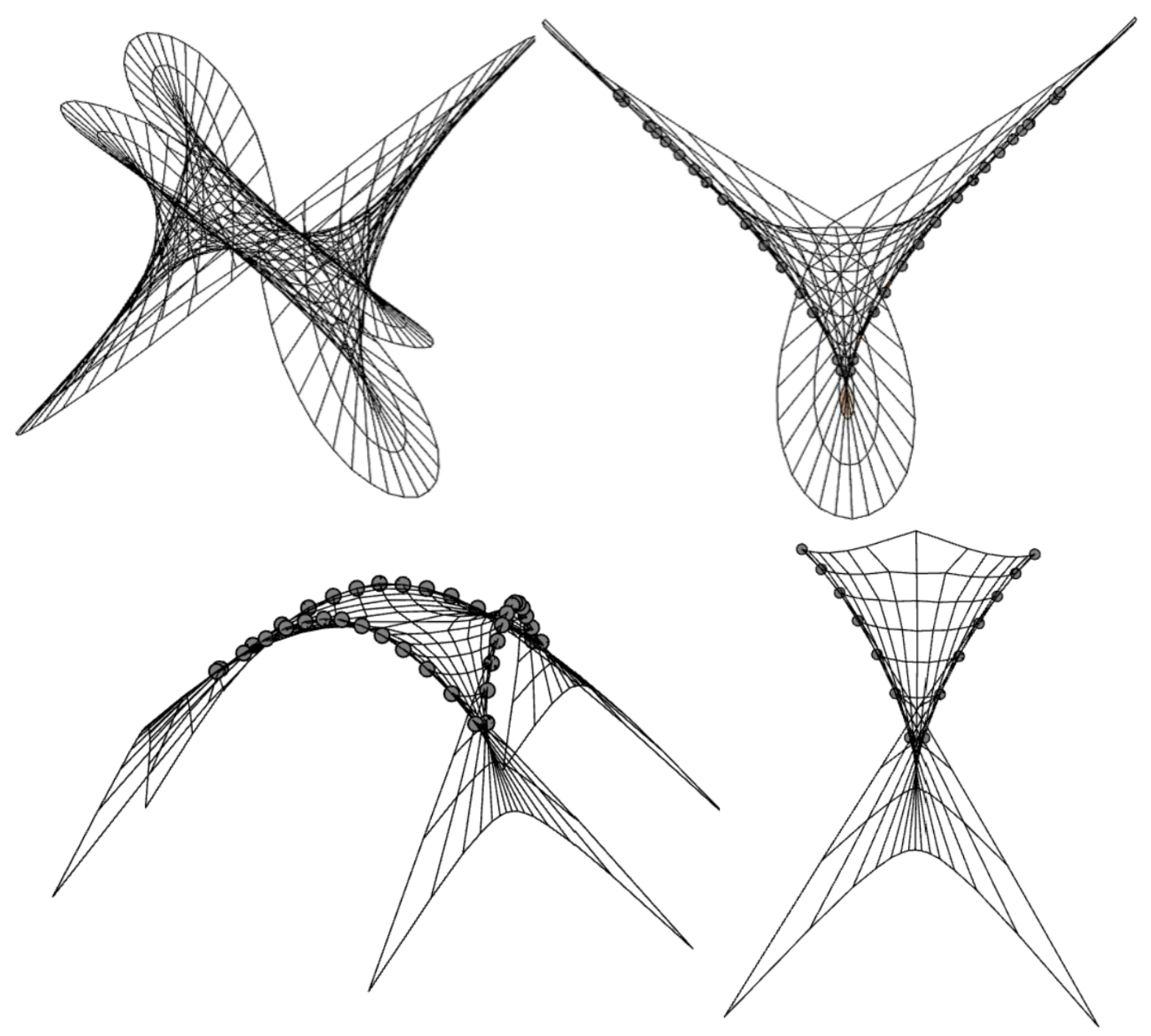

FiguRE 5. A discrete higher-order Enneper-type maximal surface in $\mathbb{R}^{2,1}$, its parallel surface at distance 20 , and a one-third piece of the parallel surface, with singular vertices marked.

Proposition 9.1. A face of a discrete CMC 1 surface $\hat{n}$ with Weierstrass-type representation in $\mathbb{S}^{2,1}$ is singular if and only if

$$
\mathcal{H}:=h_{1}^{2}+h_{2}^{2}+h_{3}^{2}-\left(h_{2}-h_{1}\right)^{2}-\left(h_{3}-h_{1}\right)^{2}-\left(h_{3}-h_{2}\right)^{2} \leq 0 .
$$

Theorem 9.1. A quadrilateral of $\hat{n}$ as in Proposition 9.1 is singular for all $\lambda$ sufficiently close to zero if the corresponding circumcircle of $g$ intersects $\mathbb{S}^{1}$ transversally. The converse holds as well under the generic assumption that $\partial_{\lambda} \mathcal{H} \neq 0$.

Proof. If the four points $g_{i}, g_{j}, g_{k}, g_{l}$ lie on a circle with radius $r \in \mathbb{R}$ and center $p \in \mathbb{C}$, the condition for $\mathcal{H}<0$ at $\lambda=0$ is

$$
\left(|p|^{2}-(r-1)^{2}\right)\left(|p|^{2}-(r+1)^{2}\right)<0 .
$$

The result follows. 

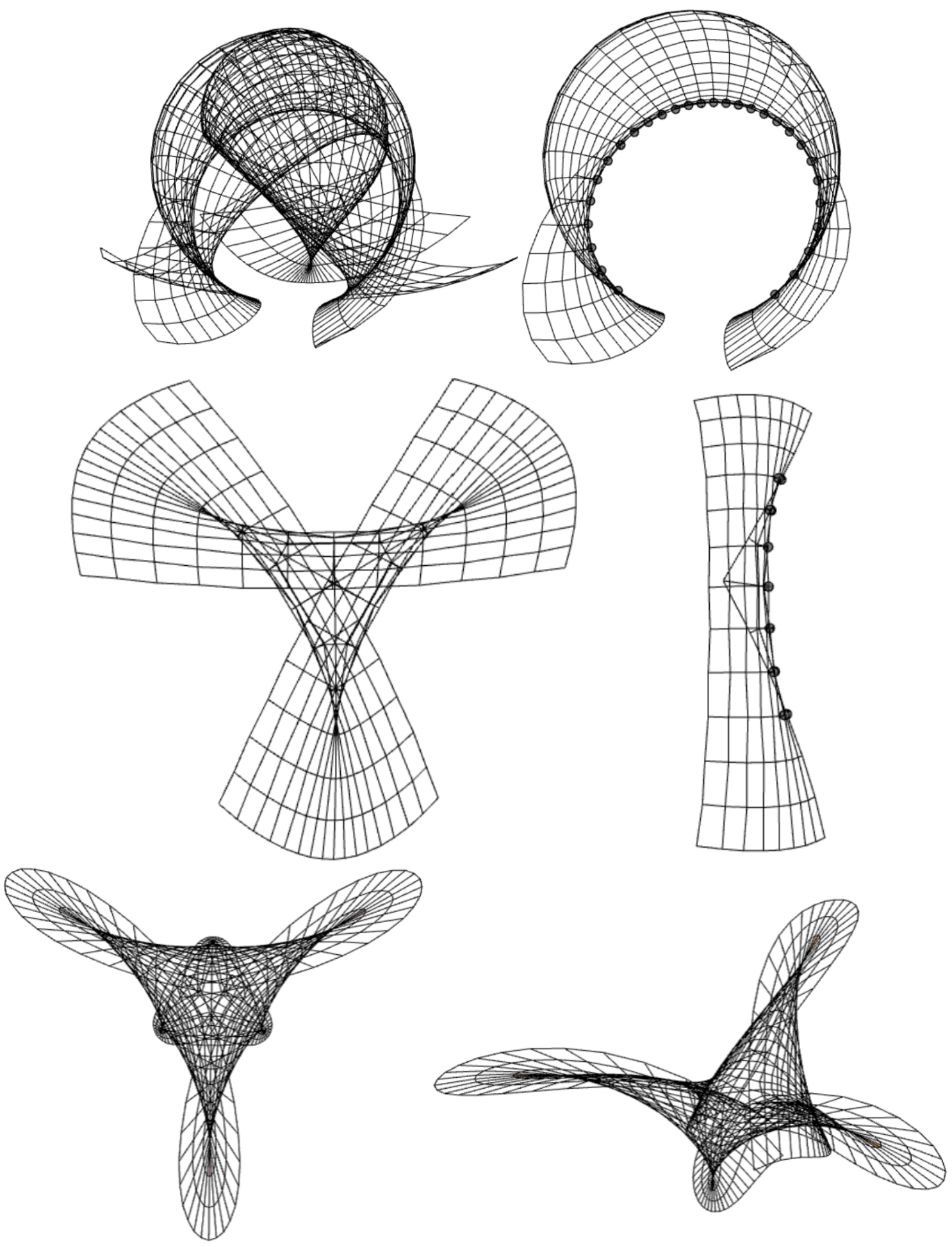

Figure 6. From top to bottom: A discrete harmonic mean curvature 1 surface in $\mathbb{S}^{2,1}$, a discrete flat surface in $\mathbb{S}^{2,1}$ and a discrete CMC 1 surface in $\mathbb{S}^{2,1}$, each shown twice. In order to draw the surfaces, we project to the hollow ball model for $\mathbb{S}^{2,1}$. (For an explanation of the hollow ball model, see [12] for example.) 


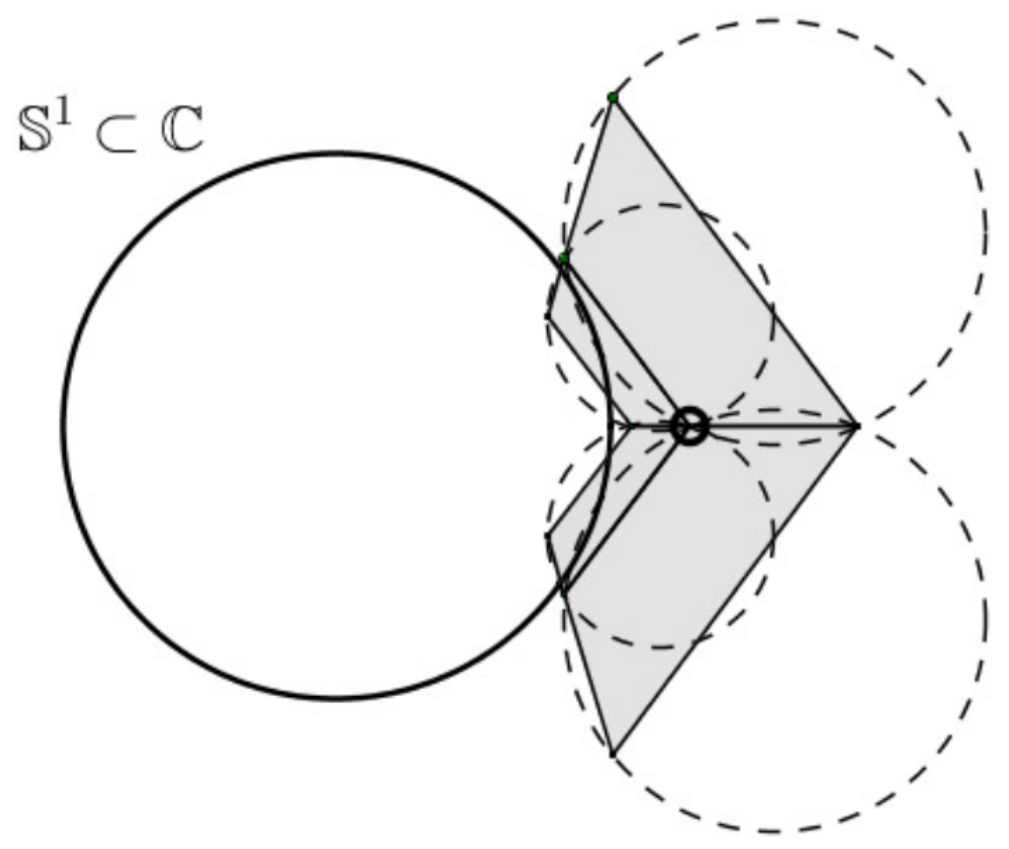

Figure 7. A counterexample to the converse in Corollary 9.1. Numerical data for a discrete holomorphic function is shown. The four faces of a discrete CMC 1 surface determined from the four gray faces above are singular faces. On the other hand, for sufficiently small $\lambda$, the marked vertex is not singular.

Theorem 9.2. Let $p_{-}, p$ and $p_{+}$be three consecutive vertices in one direction in the lattice domain of a CMC 1 surface $\hat{n}$ with Weierstrass-type representation in $\mathbb{S}^{2,1}$, with corresponding values $g_{-}, g$ and $g_{+}$for the discrete holomorphic function in the Weierstrass type representation. Under the genericity assumption $|g| \neq 1$, then $\kappa_{p_{-}} \cdot \kappa_{p p_{+}}<0$ for all $\lambda$ sufficiently close to zero if and only if exactly one of $\left|g_{-}\right|^{2}$ and $\left|g_{+}\right|^{2}$ has value less than 1 and the other has value greater than 1 .

Proof. Because the surface is CMC 1 in $\mathbb{S}^{2,1}$, we have $t=-1$. Then Equations (7) and (13) imply the result.

This theorem tells us that we will find FPS vertices roughly where $g$ (discretely) crosses $\mathbb{S}^{1}$. Because of Theorem 9.2 , we can now regard these points as singular vertices and not parabolic nor flat points. Combining Theorems 9.1 and 9.2, the following rigorous statement is immediate:

Corollary 9.1. Under the conditions of Theorem 9.2, for all $\lambda$ sufficiently close to zero, the pair of faces adjacent to the edge $p_{-} p$ are singular, or the pair of faces adjacent to the edge $p_{+}$are singular, including the possibility that all four faces are singular.

The converse of this corollary does not hold, that is, it is possible to have four singular faces (for all $\lambda$ sufficiently close to 0 ) adjacent to a given vertex that is non-singular for all $\lambda$ sufficiently close to 0 (see Figure 7). Furthermore, taking $\lambda \rightarrow 0$, the example in Figure 7 demonstrates that the converse to Theorem 8.1 also does not hold. 


\section{ACKNOWLEDGEMENTS}

The first author was partly supported by the Grant-in-Aid for Scientific Research (C) 15K04845 and (S) 24224001, Japan Society for the Promotion of Science. The second author was supported by the Grant-in-Aid for JSPS Fellows Number 26-3154, and the JSPS Program for Advancing Strategic International Networks to Accelerate the Circulation of Talented Researchers Mathematical Science of Symmetry, Topology and Moduli, Evolution of International Research Network based on OCAMI. Both authors were supported by the Grant-in-Aid for JSPS Fellows Number 26-3154, JSPS/FWF bilateral joint project "Transformations and Singularities" between Austria and Japan.

\section{REFERENCES}

[1] R. Aiyama and K. Akutagawa, Kenmotsu-Bryant type representation formulas for constant mean curvature surfaces in $\mathbb{H}^{3}\left(-c^{2}\right)$ and $\mathbb{S}_{1}^{3}\left(c^{2}\right)$, Ann. Global Anal. Geom. (1) 17 (1998), 49-75.

[2] J. A. Aledo and J. M. Espinar, A conformal representation for linear Weingarten surfaces in the de Sitter space, J. Geom. and Phys. 57 (2007), 1669-1677.

[3] A. Bobenko and U. Pinkall, Discrete isothermic surfaces, J. Reine Angew. Math., 475 (1996), $187-208$.

[4] A. Bobenko, H. Pottmann and J. Wallner, A curvature theory for discrete surfaces based on mesh parallelity, Math. Ann. 348 (2010), 1-24.

[5] A. Bobenko and Y. Suris, Discrete differential geometry, integrable structure, Graduate Textbooks in Mathematics 98, A.M.S., 2008.

[6] R. Bryant, Surfaces of mean curvature one in hyperbolic 3-space, Asterisque 154-155 (1987), $321-347$.

[7] F. E. Burstall, U. Hertrich-Jeromin and W. Rossman, Lie geometry of flat fronts in hyperbolic space, C. R. Acad. Sci. Paris, Ser. I 348 (2010), 661-664.

[8] _ Lie geometry of linear Weingarten surfaces, C. R. Acad. Sci. Paris, Ser. I 350 (2012), $413-416$.

[9] _ Discrete linear Weingarten surfaces, to appear in Nagoya Math J.

[10] F. E. Burstall, U. Hertrich-Jeromin, W. Rossman and S. Santos, Discrete surfaces of constant mean curvature, RIMS Kokyuroku 1880, 2014, 133-179.

[11] _ _ Discrete special isothermic surfaces, Geom. Dedic. 174, 2015, 1-11.

[12] S. Fujimori, Spacelike CMC 1 surfaces with elliptic ends in de Sitter 3-Space, Hokkaido Math. J. 35 (2006), 289-320.

[13] S. Fujimori, K. Saji, M. Umehara and K. Yamada, Singularities of maximal surfaces, Math. Zeit. 259 (2008), 827-848.

[14] J. A. Gálvez, A. Martínez and F. Milán, Flat surfaces in hyperbolic 3-space, Math. Ann. 316 (2000), 419-435.

[15] Complete linear Weingarten surfaces of Bryant type, a Plateau problem at infinity, Trans. A.M.S. 356 (2004), 3405-3428.

[16] U. Hertrich-Jeromin, Transformations of discrete isothermic nets and discrete cmc-1 surfaces in hyperbolic space, Manusc. Math. 102 (2000), 465-486.

[17] _ Introduction to Möbius differential geometry, London Mathematical Society Lecture Note Series 300, 2003.

[18] U. Hertrich-Jeromin, T. Hoffmann and U. Pinkall, A discrete version of the Darboux transform for isothermic surfaces, Oxf. Lect. Ser. Math. Appl. 16 (1999), 59-81.

[19] T. Hoffmann, W. Rossman, T. Sasaki, M. Yoshida, Discrete flat surfaces and linear Weingarten surfaces in hyperbolic 3-space, Trans. A.M.S. 364 (2012), 5605-5644.

[20] S. Izumiya and K. Saji, The mandala of Legendrian dualities for pseudo-spheres in LorentzMinkowski space and "flat" spacelike surfaces, J. of Singularities 2 (2010), 92-127. 
[21] Y. Kinoshita and W. Rossman, Isothermicity of discrete surfaces in the Euclidean and Minkowski 3-spaces, OCAMI publications 3 (2010), 1-13.

[22] O. Kobayashi, Maximal surfaces in the 3-dimensional Minkowski space $\mathbb{L}^{3}$, Tokyo J. Math. 6 (1983), 297-309.

[23] M. Kokubu and M. Umehara, Orientability of linear Weingarten surfaces, spacelike CMC1 surfaces and maximal surfaces, Math. Nachr. 284, 1415 (2011), 1903-1918.

[24] Y. Ogata and M. Yasumoto, Construction of discrete constant mean curvature surfaces in Riemannian spaceforms and applications, preprint.

[25] W. Rossman, Discrete Constant Mean Curvature Surfaces via Conserved Quantities, Math for Industry GCOE Lecture Note, Kyushu Univ., Vol. 25 (2010), 1-130.

[26] _ Isothermic surfaces in Möbius and Lie sphere geometries, Rokko Lec. Series Math. 22 (2014), 1-138.

[27] K. Teramoto, Parallel and dual surfaces of cuspidal edges, Diff. Geom. Appl. 44 (2016), 52-62.

[28] K. Teramoto, Principal curvatures of wave fronts, preprint.

[29] M. Umehara, K. Yamada, Complete surfaces of constant mean curvature 1 in the hyperbolic 3space, Annals of Math. 137 (1993), 611-638.

[30] M. Umehara, K. Yamada, Maximal surfaces with singularities in Minkowski space, Hokkaido Math. J. 35 (2006), 13-40.

[31] W. Wunderlich, Zur Differenzengeometrie der Flachen konstanter negativer Krummung, Osterreich. Akad. Wiss. Math.-Nat. Kl. S.-B. IIa., 160 (1951), 39-77.

[32] M. Yasumoto, Discrete maximal surfaces with singularities in Minkowski space, Diff. Geom. Appl. 43 (2015), 130-154. .

(W. Rossman) Department of mathematics, Faculty of science, Kobe University

RokKODAI-ChO 1-1, NADA-KU, Kobe, 657-8501, JaPAN

E-mail address: wayne@math.kobe-u.ac.jp

(M. Yasumoto) Department of mathematics, Faculty of science, Kobe University ROKKODAI-CHO 1-1, NADA-KU, KoBe, 657-8501, JAPAN

Current address, M. Yasumoto: Institut für Mathematik, Universität Tübingen, Auf der Morgenstelle 10, 72076 Tübingen, Germany

E-mail address: myasu@math.kobe-u.ac.jp 